\title{
DISCUSSION
}

\section{A generalized procedure for predicting optimal lower bound break-out factors of strip anchors}

\author{
P. K. BASUDHAR and D. N. SINGH (1994) Géotechnique 44, No. 2, 307-318
}

E. J. Murray, Murray Rix Geotechnical, and J. D. Geddes, formerly University of Wales College of Cardiff, now Consultant

The Authors describe the development of lower bound limit analysis solutions for horizontal and vertical strip anchors in a cohesionless medium. It is interesting to compare their lower bound predictions with upper bound solutions and experimental evidence.

In simplistic terms a valid upper bound requires a compatible and continuous failure mechanism to be determined while a lower bound requires the formulation of a statically admissible stress field to be continuous everywhere and nowhere to exceed the yield criterion.

The proofs of the upper and lower bound limit analysis theorems are outlined in a number of publications (e.g. Chen, 1975). The theorems enable the true theoretical failure load of an idealized soil to be bracketed, and in some cases to be defined if there is convergence of the bound solutions. Although theorems have been developed which yield close bounds to the collapse loading of a number of problems involving an idealized material which obeys an associated flow rule (i.e. the angle of shearing resistance $\phi$ equals the angle of dilation $\Psi$ and the normality principle holds true), only restricted theorems have been developed for the more general class of material which deforms under non-associated flow conditions $(\phi>\Psi)$. Frictional soils would be more closely represented by a non-associated flow rule. It is necessary to determine the minimum upper bound solution and the maximum lower bound solution in order to assess as accurately as possible the collapse or failure condition for anchors embedded in the idealized material.

The Authors' analyses for smooth anchors yield true lower bound solutions. However, this is not the case for anchors with interface friction, as such friction gives rise to energy dissipation and the solutions cannot be shown to be true lower bounds. Nevertheless, from a practical point of view, analyses taking interface friction into account are normally considered as providing a lower limit to the yield load for a material obeying an associated flow rule.
For horizontal anchors, Murray (1977) and Murray \& Geddes (1987) show that the minimum upper bound solution for a strip anchor is given by

$$
\frac{P}{\gamma B H}=1+\frac{H}{B} \tan \phi
$$

This is in agreement with Vermeer \& Sutjiadi (1985). To comply more closely with observed experimental failure patterns, Vermeer \& Sutjiadi modified their solutions for strip and rectangular anchors for a cohesionless material where $\phi>\Psi$ and the constant volume friction angle $\phi_{\mathrm{cr}}$ is taken into account. Although these latter solutions provide a sensible method of assessing the failure loads and are used in the Paper, they do not comply with the requirements of upper bound solutions. In particular, the equation for a rectangular anchor does not have a continuous acceptable displacement field at the corners.

The minimum upper bound solution for a rectangular anchor is given by (Murray \& Geddes, 1987)

$$
\begin{aligned}
\frac{P}{\gamma B H}= & 1+\frac{H}{B} \tan \phi \\
& \times\left(1+\frac{B}{L}+\frac{\pi}{3} \frac{H}{L} \tan \phi\right)
\end{aligned}
$$

This solution reduces to equation (19) for the case of a strip anchor with $L=\infty$.

The upper bound solution of equation (19) for $L=\infty$ and the Authors' lower bound solution for a smooth plate converge, indicating that equation (19) provides a simple solution to the true failure load for an idealized soil obeying an associated flow rule. Although an upper bound solution for a material obeying an associated flow rule is also an upper bound for a material obeying a non-associated flow rule (i.e a real soil), as shown in Fig. 12 there is a close correlation between the experimental evidence for smooth plates of $L / B \geqslant 5$ in medium dense sand and the theoretical predictions. Murray \& Geddes (1987) have also shown that there is close correlation between the experimental uplift capacity of 


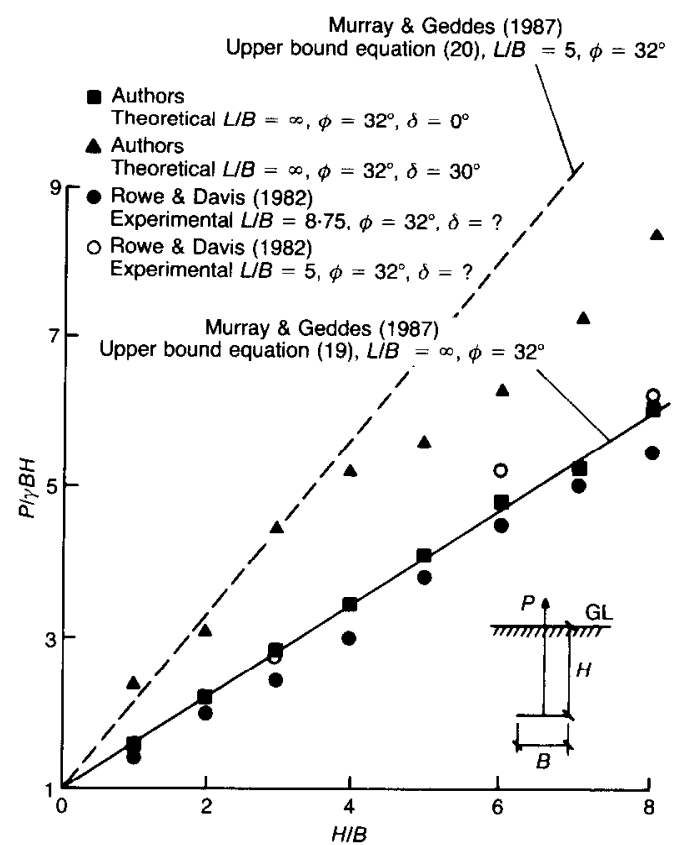

Fig. 12. Variation of break-out factor with embedment ratio for horizontal anchors

smooth anchors and the upper bound solution for very dense sand.

Analysis of various compatible failure mechanisms (both symmetrical and asymmetrical) in determining the minimum upper bound solution indicates that the symmetry of the anchor uplift problem results in a mechanism with no movement on the anchor face, whether the anchor is smooth or rough. According to Drucker (1954) the solution for no relative movement would constitute an upper bound for a smooth or a rough plate. Conversely the lower bound solution with no interface friction would be a lower bound for a plate with finite friction. The predictions of the Authors, taking interface friction into account, exceed the predictions of equation (19) as shown in Fig. 12. Thus Drucker's theorem is in conflict with the anchor uplift problem although, strictly as already outlined, any attempt to take interface friction into account in a lower bound solution would invalidate its being considered as a true lower bound in accordance with the limit analysis theorems.

As shown by Murray \& Geddes (1987), experimental evidence is also in agreement with an increase in uplift loading with an increase in interface friction for the horizontal anchor problem. It seems appropriate to treat equation (19) as yielding an upper bound for a smooth plate.

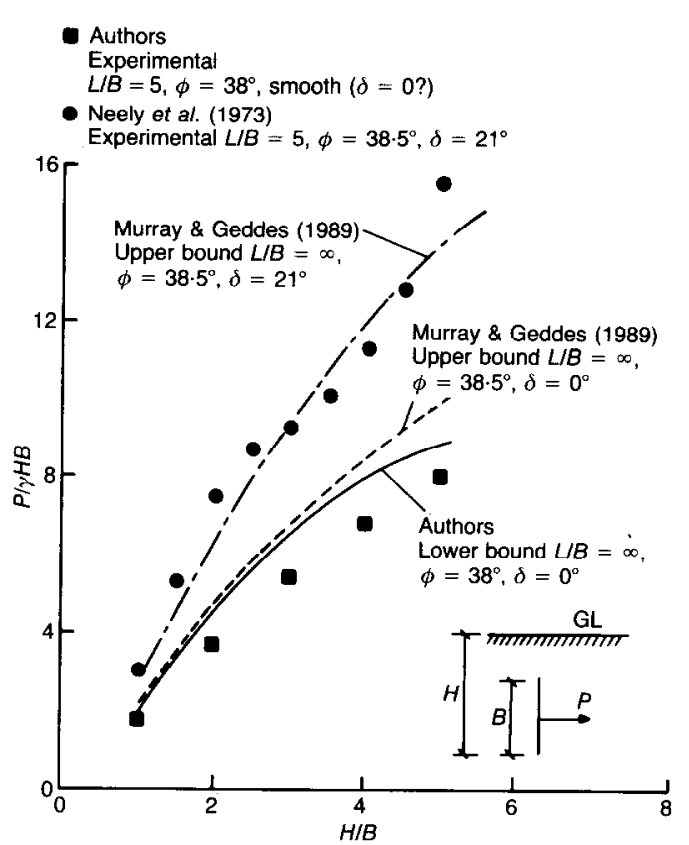

Fig. 13. Variation of break-out factor with embedment ratio for vertical anchors

However, as shown in Fig. 12, equation (20) indicates a substantial increase in uplift capacity for rectangular anchors. This equation would again be an upper bound for smooth and rough anchors. The analyses and experimental evidence indicate that it is essential to take proper account of the roughness of the anchor plate and its $L / B$ ratio when attempting to predict anchor uplift capacity.

Figure 13 compares theoretical upper and lower bound solutions for a vertical strip anchor and experimental results. Of particular interest is the close correlation between the lower bound solutions of the Authors for a smooth strip anchor with $\phi=38^{\circ}$ and the upper bound solutions of Murray \& Geddes (1989) for a similar anchor in a cohesionless medium with $\phi=38.5^{\circ}$. This suggests that the failure loading for a material obeying an associated flow rule is defined or closely bracketed. The upper bound solution would be expected to overpredict the results for a real soil with $\phi>\Psi$ and this is supported by the experimental results reported by the Authors as shown in Fig. 13. However, further experimental results in very dense sand (Murray \& Geddes, 1989) suggest that the theoretical upper bound can underpredict experimental results although it can provide a reasonable assessment.

The upper bound solution for a plate with interface friction $\delta=21^{\circ}$ in a similar cohesionless 
material $\left(\phi=38.5^{\circ}\right)$ indicates an increase in anchor capacity for a rough plate; this compares favourably with the results of Neely, Stuart \& Graham (1973) as shown in Fig. 13. The upper bound with a frictional interface is valid provided the solution is developed in accordance with Drucker (1954). Again, in agreement with the Authors, the importance of taking interface friction into account is shown.

The correspondence between our upper bound limit analysis solutions for horizontal and vertical anchors and the Authors' lower bound solutions is encouraging, and the reasonable correlation with experimental evidence lends more confidence in the use of the techniques for predicting anchor capacity. Palmer (1966) suggests from experimental evidence that the Coulomb yield criterion appears to represent a lower bound yield criterion for real soils. This does not mean that a lower bound statically admissible stress field solution using the Coulomb criterion is a lower bound for a real soil, but it adds some weight to the use of the lower bound approach. Thus, although an upper bound solution for a material obeying an associated flow rule is also an upper bound for a material obeying a non-associated flow condition, where there is convergence of upper and lower bound solutions, reasonable correlation with experimental evidence is perhaps not so surprising. However, there is experimental evidence to indicate that other factors affect anchor capacity which are not taken into account in limit analysis solutions (Geddes \& Murray, 1991) and these obviously influence any comparison between theory and experiment.

\section{Authors' reply}

The method of analysis finds an equilibrium distribution of stress in the significant zone under consideration and balances the applied loads on the stress boundary without violating the yield condition anywhere. As such, it may be considered to be a lower bound. However, it would be more appropriate to study the extensibility of the stress field to check whether the solution obtained is a partial solution or a true lower bound. Another aspect that needs attention is the effect of the mesh geometry and the mesh size on the solution.

When the coefficient of friction is zero, or if there is no relative motion or separation at the frictional interfaces, the limit theorems of plasticity would apply.

Chen (1975) states that any set of loads which will not cause collapse of an assemblage of elastic-plastic bodies with frictional interfaces when all coefficients of friction at the interfaces are zero, will not produce collapse with any values of the coefficients. Thus, if the anchor interface is taken to be a plane of principal stress, the estimated capacity is safe for a smooth anchor and hence for an anchor with finite friction. Our study highlights the need to include the effect of anchor roughness on anchor capacity; the predictions made by using the proposed approach arc in good agreement with experimental observations of Rowe \& Davis (1982) and are lower than the experimentally observed failure load. For finite friction with friction angle $\delta$, Chen has suggested the use of the concept of interface cement' which is composed of a soil-like cohesionless material with a friction angle $\phi=\delta$ obeying the Coulomb yield criterion and its associated flow rule. Thus, the limit theorems are applicable to the modified body with cemented interfaces. As the state of stress as predicted by the proposed method does not violate the condition (equation (13)) and at the interface it satisfies $\tau_{i j} \leq \mu \sigma_{i j}$ (equation (10)), a safe state of stress exists in the friction problem. Imposition of these interface constraints implies that at the limiting state there is no slip between the soil and the anchor at the interface.

The points raised regarding the nature of the deviation of our solution from the upper bound solution for vertical rough anchors given in the Paper are noted; further study on this aspect is needed.

\section{REFERENCES}

Chen, W.-F. (1975). Limit analysis and soil plasticity. In Developments in geotechnical engineering, vol. 7, pp. 41-45. Amsterdam: Elsevier.

Drucker, D. C. (1954). Coulomb friction, plasticity and limit loads. Trans. Am. Soc. Mech. Engrs 76, 71-74.

Geddes, J. D. \& Murray, E. J. (1991). Passive inclined anchorages in sand. J. Geotech. Engng Am. Soc. Civ. Engrs 117, No. 5, 810-814.

Murray, E. J. (1977). The passive resistance of anchorages in sand. PhD thesis, University of Wales Institute of Science and Technology.

Murray, E. J. \& Geddes, J. D. (1987). Uplift of anchor plates in sand. J. Geotech. Engng Am. Soc. Civ. Engrs 113 , No. 3, 202-215.

Murray, E. J. \& Geddes, J. D. (1989). Resistance of passive inclined anchors in cohesionless medium. Géotechnique 39, No. 3, 417-431.

Neely, W. J., Stuart, J. G. \& Graham, J. (1973). Failure loads of vertical anchor plates in sand. J. Soil Mech. Fdns Div. Am. Soc. Civ. Engrs 99, SM9, 669-685.

Palmer, A. C. (1966). A limit theorem for materials with non-associated flow laws. J. Méc. 5, No. 2, 127-222.

Rowe, R. K. \& Davis, E. H. (1982). The behaviour of anchor plates in sand. Géotechnique 32, No. 1, 2541.

Vermeer, P. A. \& Sutjiadi, W. (1985). The uplift resistance of shallow embedded anchors. Proc. 1lth Int. Conf. Soil Mech., San Francisco 3, 1635-1638. 